\title{
Surface plasmon-polariton model in polygonal chiral thin films
}

\author{
F Babaei and V Bekdelo \\ Department of Physics, Faculty of Science, Qom University, Qom, Iran
}

E-mail: fbabaei@qom.ac.ir

(Received 05 December 2018 ; in final form 26 July 2019)

\begin{abstract}
In this research, the surface plasmon-polariton modes at interface of a metal and a polygonal chiral thin film in Kretschman configuration have been theoretically studied. With the depiction of optical absorption spectra for P-linear polarized incident light, the surface plasmonic modes from the waveguide modes have been distinguished. The effect of structural parameters, such as the thickness of polygonal chiral thin film, the thickness of the metallic thin film and the growth angle of chiral columns, on the propagation of plasmonic modes has been investigated. The results show that more than one plasmonic mode can be excited at the interface of a polygonal chiral thin film and a metal.
\end{abstract}

Keywords: surface plasmon- polariton, polygonal chiral thin film

For full article, refer to the Persian section 